\title{
Influência do Tempo de Coleta e Metodologias sobre a Digestibilidade e o Valor Energético de Rações para Aves ${ }^{1}$
}

\section{Paulo Borges Rodrigues ${ }^{2}$, Ricardo de Souza Martinez ${ }^{3}$, Rilke Tadeu Fonseca de Freitas ${ }^{2}$, Antônio Gilberto Bertechini², Elias Tadeu Fialho²}

\begin{abstract}
RESUMO - Em um ensaio de digestibilidade, avaliou-se o método de coleta total de excretas e o método do óxido crômico como indicador. Determinou-se a energia metabolizável aparente corrigida pelo nitrogênio retido (EMAn) e os coeficientes de digestibilidade aparente da matéria seca (CDAMS) de uma ração à base de milho e farelo de soja, contendo 19\% de PB e 3.100 kcal de EM/kg. Foram

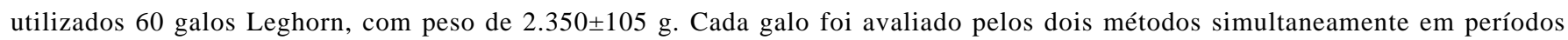
consecutivos. Utilizou-se um delineamento inteiramente casualizado, com parcelas subdivididas, para as duas metodologias avaliadas. Com o uso do óxido crômico, os tratamentos foram definidos em um arranjo fatorial 4x5 (quatro níveis de óxido crômico, 0,2; 0,4; 0,6 e 0,8\%, e cinco tempos de coleta de excretas, 1 a 5 dias), totalizando 20 tratamentos na parcela (galo), em dois períodos consecutivos (subparcelas), com três repetições por período. Na coleta total de excretas, os tratamentos foram definidos como cinco tempos de coleta ( 1 a 5 dias) na parcela e dois períodos consecutivos na subparcela, com 12 repetições por período. O uso dos períodos consecutivos de coleta não afetou a EMAn nem o CDAMS. No método de coleta total, os valores de EMAn e CDAMS atingiram um platô acima de dois dias de coleta, comprovando que o tempo de coleta pode ser reduzido para três dias sem comprometer os valores determinados. Os resultados indicaram interação dos níveis de óxido crômico na ração e do tempo de coleta para os valores de EMAn e CDAMS. Concluiu-se que três dias de coleta são suficientes para determinar a EMAn e o CDAMS de rações à base de milho e farelo de soja. Com três dias de coleta e com o uso de $0,665 \%$ de óxido crômico na ração, foi possível determinar valores semelhantes de EMAn e CDAMS nas duas metodologias.
\end{abstract}

Palavras-chave: coleta total, energia metabolizável, galos adultos, matéria seca, óxido crômico

\section{Effect of Collection Time and Methodologies on the Digestibility and Energy Value of Poultry Diets}

\begin{abstract}
A digestibility assay were carried out to evaluate, in consecutives periods, the total excreta collection method and the chromium oxide method as a marker. Apparent metabolizable energy corrected by nitrogen retention (AMEn) and aparent digestibility of dry matter coefficient (ADDMC) of broiler diet based in a ground corn and soybean meal containing $19 \%$ crude protein and 3.100 kcal $\mathrm{ME} / \mathrm{kg}$. Sixty adult Leghorn cockerels weigh, in average, $2.350 \pm 105 \mathrm{~g}$ were used. Each cockerel participates in the evaluation of the two methods simutannialy in consecutives periods. A split plots experimental design experiment was used to evaluate the two métodhs. In the chromium oxid method the treatments were defined as a $4 \times 5$ factorial arrangemente (chromium oxide levels $-0.2,0.4,0.6$ and $0.8 \%$ and collection time - 1 to 5 days), totalizing 20 treatments in the plot, in two continuous periods (sub-plots), with three replicates by period. In the total excreta collection method the treatments were defined as five collection times ( 1 to 5 days) in the plot and the two continuous periods in the subplot with twelve replicates per period. The use of continuous periods did not affect AMEn or ADDMC. In the total excreta collection method the values of AMEn and ADDMC reached a plateau after two days of collection, showing that the collection time could be reduced by three days without affecting the determinated values. The data showed interaction between chromium oxide levels with collection times for the AMEn and ADDMC. Three days of excreta collection were enough to determine with accuracy both AMEn and ADDMC of the diets based on ground corn and soybean meal. The use of three days of collection and $0.665 \%$ of chromium oxide in the diet was possible to determine similar values for AMEn and ADDMC in the two methods.
\end{abstract}

Key Words: adult cockerels, chromium oxide, dry matter, metabolizable energy, total collection

\section{Introdução}

Metodologias para se avaliar a digestibilidade dos nutrientes em rações para aves têm sido objeto de vários estudos realizados nas três últimas décadas,
(Sibbald, 1976; Farrel, 1981; Hartel, 1986; Albino, 1992; McNab, 1996; Zelenka, 1997). Entre os vários métodos empregados na determinação dos valores energéticos dos alimentos para aves, destacam-se o tradicional de coleta total de excretas (Sibbald \&

\footnotetext{
${ }_{1}^{1}$ Parte da dissertação de Mestrado do segundo autor.

2 Professores do DZO/UFLA - Pesquisadores bolsistas do CNPq (pborges@ufla.br).

3 Zootecnista, MSc. (ricardo@vaccinar.com.br).
} 
Slinger, 1963), o da alimentação precisa (Sibbald, 1976) e o método rápido (Farrel, 1978). Entretanto, o método de coleta total de excretas é o mais utilizado para a determinação da energia metabolizável aparente corrigida (EMAn).

O uso do óxido crômico na dieta das aves, quando em experimentos, permite estimar a produção de matéria seca (MS) fecal e o fluxo de MS nas diferentes partes do trato gastrointestinal, evitando-se a coleta total de excretas, tradicionalmente usada na digestibilidade in vivo dos alimentos. Nesta metodologia, normalmente se faz uma coleta parcial em locais mais limpos, ou seja, isentos de penas ou restos de ração, tornando a fase experimental menos trabalhosa e mais segura. No entanto, este método é muito criticado, pois a determinação do cromo, possivelmente por falta de padronização no preparo das amostras em laboratório, é sujeita a grandes variações, o que resulta em dados variados, limitando sua utilização.

Diversos estudos relatam comparações entre métodos de determinação dos valores de EMA. Sibbald (1960) encontrou valores mais precisos quando utilizou óxido crômico como indicador fecal, enquanto Potter (1972) concluiu que os melhores resultados foram obtidos pelo método de coleta total de excretas. De acordo com Tenesaca \& Sell (1981), 24 horas de coleta foram insuficientes para esvaziar o TGI quando se utilizou sílica diluída em uma ração basal. Semelhantes resultados foram encontrados por Parsons et al. (1982), que propuseram o período de 30 horas de coleta como o mais preciso.

A extensão do período de coleta de excretas depende do ingrediente a ser testado. Recomenda-se o uso de períodos mais longos (48 horas) para coleta de excretas de aves alimentadas com ingredientes que propiciem taxa de passagem mais lenta (Kessler \& Thomas, 1981). Da mesma forma, Fialho et al (1985), utilizando suínos em estudo de metabolismo, encontraram menores erros-padrão da média com o aumento do período de dias de coleta, indicando uma maior precisão das estimativas para períodos maiores. No entanto, deve-se considerar que, no método tradicional, as aves passam por um período de adaptação às dietas testes, normalmente de cinco dias, seguidos de cinco dias de coleta, o que pode ser desnecessário. Objetivou-se, com esse trabalho: a) determinar o melhor período de coleta para determinação precisa da EMAn e do CDAMS, pelo método tradicional de coleta total e pelo método do indicador óxido crômico; b) avaliar os níveis de inclusão do indicador óxido crômico com relação a precisão das estimativas e; c) comparar os valores obtidos nas duas metodologias.

\section{Material e Métodos}

Foi conduzido um ensaio de digestibilidade no setor de Avicultura do Departamento de Zootecnia da Universidade Federal de Lavras - UFLA, onde se avaliou, ao mesmo tempo, o método de coleta total de excretas e o uso do óxido crômico como indicador, para determinação dos valores de EMAn e CDAMS de uma ração à base de milho e farelo de soja, formulada para conter $19 \%$ de PB e $3100 \mathrm{kcal}$ de EM/kg (Tabela 1). Foram utilizados 60 galos Leghorn adultos, com peso de $2.350 \pm 105 \mathrm{~g}$, distribuídos individualmente em gaiolas de estudos metabólicos e submetidos aos mesmos tratamentos experimentais em dois períodos consecutivos.

Como cada galo recebeu o mesmo tratamento em períodos consecutivos, utilizou-se um delineamento experimental inteiramente casualizado, com parcela subdividida para as duas metodologias avaliadas. Para a metodologia do óxido crômico, os tratamentos foram definidos em um arranjo fatorial $4 \times 5$ (quatro níveis de óxido crômico, 0,2; 0,4; 0,6 e 0,8\% e cinco tempos de coleta de excretas, 1 a 5 dias), totalizando 20 tratamentos na parcela (galo), em dois períodos consecutivos, que constituiu-se na subparcela, com três repetições por período. Para a metodologia de coleta total de excretas, os tratamentos foram definidos como cinco tempos de coleta ( 1 a 5 dias) na parcela e dois períodos consecutivos na subparcela, com 12 repetições por período, sendo cada galo a unidade experimental. Assim, uma vez quantificado o consumo e o total excretado, e, como as metodologias diferem apenas nos cálculos dos valores energéticos e dos coeficientes de digestibilidade, sem interferência de uma sobre a outra, foi possível avaliar, simultaneamente, os valores determinados em cada uma delas utilizando o mesmo galo na unidade experimental.

Os valores de EMAn e dos CDAMS foram determinados pelas fórmulas descritas por Matterson et al. (1965), utilizando-se, na metodologia de coleta total de excretas, as fórmulas:

Energia bruta (EB) ingerida - EB excretada

$\operatorname{EMA}(\mathrm{kcal} / \mathrm{kg}$ de MS $)=$

MS ingerida 
Tabela 1 - Composição centesimal e nutritiva das rações experimentais

Table 1 - Centesimal and nutritive composition of the experimental diets

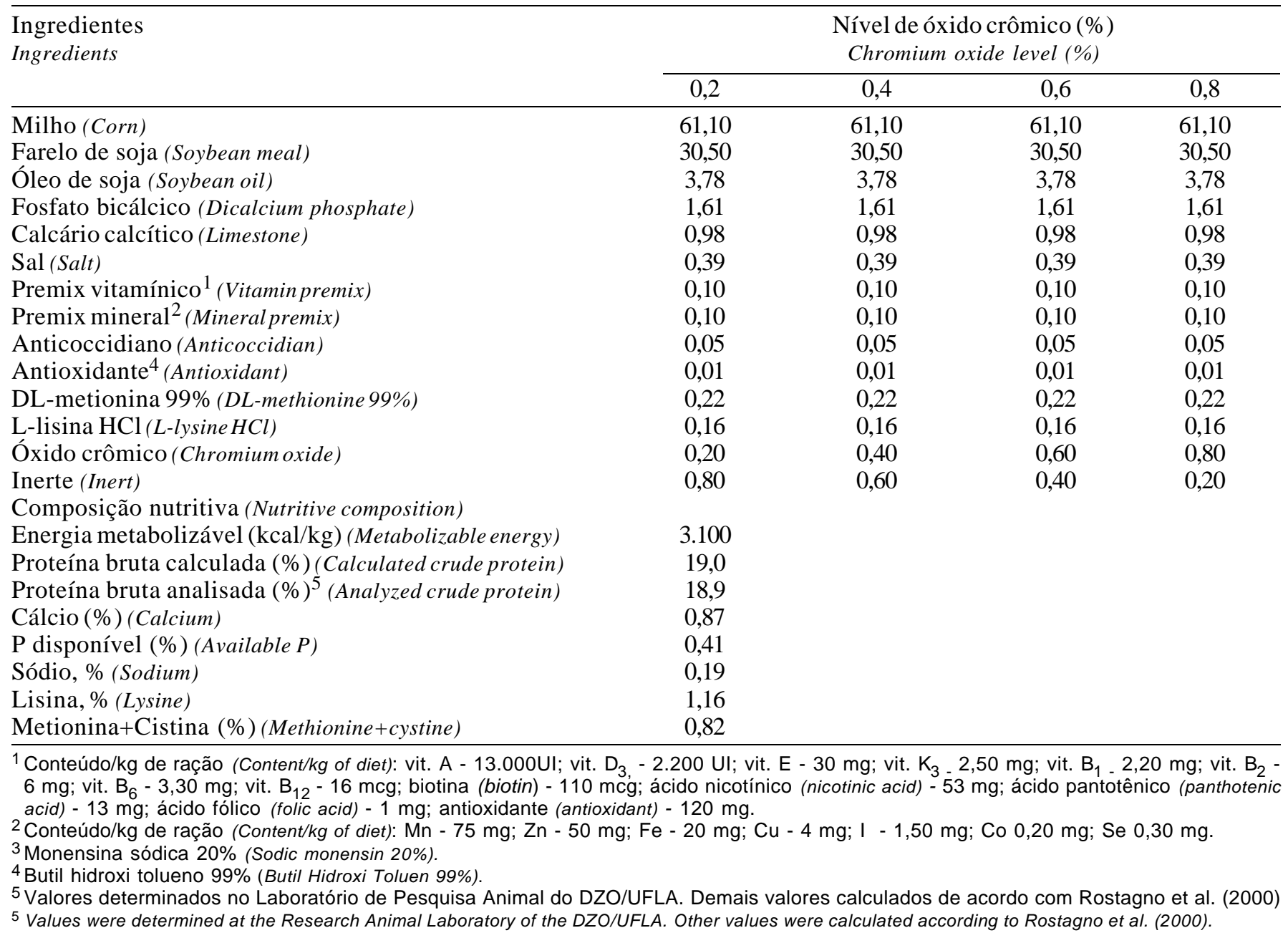

EB ingerida - $($ EB excretada + $8,22 \times \mathrm{BN})$

EMAn $(\mathrm{kcal} / \mathrm{kg}=$ de MS)

MS ingerida

$\mathrm{BN}=$ balanço de nitrogênio $=\mathrm{N}$ ingerido $-\mathrm{N}$ excretado

$$
\operatorname{CDAMS}(\%)=\frac{\text { MS ingerida }- \text { MS excretada }}{\text { MS ingerida }} \times 100
$$

Para a metodologia do óxido crômico, o cálculo da EMAn e do CDAMS foi realizado por intermédio do fator de indigestibilidade (FI) do cromo, pelas fórmulas:

FI = concentração de cromo na ração/concentração de cromo na excreta
EMA $(\mathrm{kcal} / \mathrm{kg}$ de MS $)=\mathrm{EB}$ da dieta $-(\mathrm{EB}$ das excretas $\mathrm{x}$ FI)

EMAn $(\mathrm{kcal} / \mathrm{kg}$ de MS $)=\mathrm{EB}$ da dieta $-[(\mathrm{EB}$ da excreta $\mathrm{x}$ FI $)+8,22 \times \mathrm{BN}]$

$$
\mathrm{BN}=\mathrm{N} \text { da dieta }-(\mathrm{N} \text { da excreta } * \mathrm{FI})
$$

As aves foram alojadas em gaiolas metálicas em uma sala utilizada para ensaios de metabolismo, com ambiente parcialmente controlado, e passaram inicialmente por um período de cinco dias de adaptação às dietas experimentais, seguidos pelos dois períodos consecutivos de coleta, recebendo água e ração à vontade durante todo o período experimental.

Em cada tratamento, as excretas foram coletadas e acondicionadas em sacos plásticos devidamente 
identificados, retirando-se, após o período experimental, alíquotas de até $400 \mathrm{~g}$ para as análises laboratoriais. A determinação da matéria seca e do nitrogênio da ração e das excretas foi realizada conforme os métodos descritos pela AOAC (1990). A energia bruta foi determinada em bomba calorimétrica tipo Parr e o cromo, analisado segundo a metodologia descrita por Silva (1990). As análises foram feitas utilizando-se a mesma amostra de excretas para as duas metodologias, pois, uma vez que o indicador óxido crômico é inerte, não há possibilidade de ter influenciado na determinação dos valores por meio da metodologia de coleta total de excretas.

As análises estatísticas foram realizadas emrpegando-se o pacote computacional SISVAR (Ferreira, 2000). Aplicou-se a regressão polinomial para os níveis de cromo e o modelo descontínuo (Braga, 1983), quando pertinente, para os dias de coleta. Vale ressaltar que não foi de interesse prático e não foi um dos objetivos estudar, neste trabalho, o efeito do número de dias de coleta por análise de regressão. Objetivou-se apenas definir um número de dias para melhor procedimento de coleta de excretas.

\section{Resultados e Discussão}

$\mathrm{Na}$ Tabela 2, estão apresentados os valores energéticos (EMAn) e os coeficientes de digestibilidade (CDAMS) determinados pelo método tradicional de coleta total de excretas, em diferentes tempos de coleta. A utilização dos períodos consecutivos de coleta não teve influência $(\mathrm{P}>0,05)$ sobre os valores de EMAn ou CDAMS determinados. Com relação ao tempo de coleta, verificou-se que tanto os valores de EMAn quanto os CDAMS atingiram um platô $(\mathrm{P}<0,01)$ acima do segundo dia de coleta (Figuras 1 e 2). O menor valor do erro-padrão da estimativa foi obtido aos três dias de coleta (Tabela 2), constatando-se que um período de três dias de coleta é suficiente para determinação da EMAn de forma confiável, o que confirma a afirmativa de Schang \& Hamilton (1982) de que é necessário um período mínimo de 48 horas de coleta.

No últimos anos, vários autores vêm utilizando cinco dias de coleta como padrão no método tradicional de coleta total de excretas (Albino et al., 1992; Nascimento et al., 1998; Vieites et al., 2000; Rodrigues et al., 2001, 2002), sendo que Furlan et al. (1998) utilizaram seis dias de coleta na determinação de valores energéticos de alguns alimentos para codornas. No entanto, o uso de cinco dias demanda tempo,
Tabela 2 - Coeficientes de digestibilidade da matéria seca (CDAMS) e energia metabolizável aparente corrigida pelo nitrogênio retido (EMAn), determinados em diferentes tempos de coleta pela metodologia de coleta total de excretas, e seus respectivos erros-padrão (EP)

Table 2 - Dry matter digestibility coefficients (DMDC) and apparent metabolizable energy corrected by retained nitrogen (AMEn) in different collection times by the total collection methodology with your respective standard errors (SE)

Tempo de CDAMS $(\%) \pm(\mathrm{EP})^{1}$ EMAn $(\mathrm{kcal} / \mathrm{kg}) \pm(\mathrm{EP})^{1}$ coleta $\quad D M D C \pm(S E) \quad A M E n \pm(S E)$

Collection

period

\begin{tabular}{lll}
\hline 1 dia (day) & $73,9(0,63)$ & $3599(17)$ \\
2 dias (days) & $71,0(0,43)$ & $3530(12)$ \\
3 dias (days) & $70,4(0,37)$ & $3520(10)$ \\
4 dias (days) & $70,9(0,50)$ & $3536(12)$ \\
5 dias (days) & $70,4(0,49)$ & $3513(14)$ \\
Média (Mean) & $71,3(2,7)$ & $3540(6,5)$ \\
\hline
\end{tabular}

${ }^{1}$ Resposta linear com platô - LRP $(P<0,01)$.

${ }^{1}$ Linear response plateau $-L R P(P<.01)$.

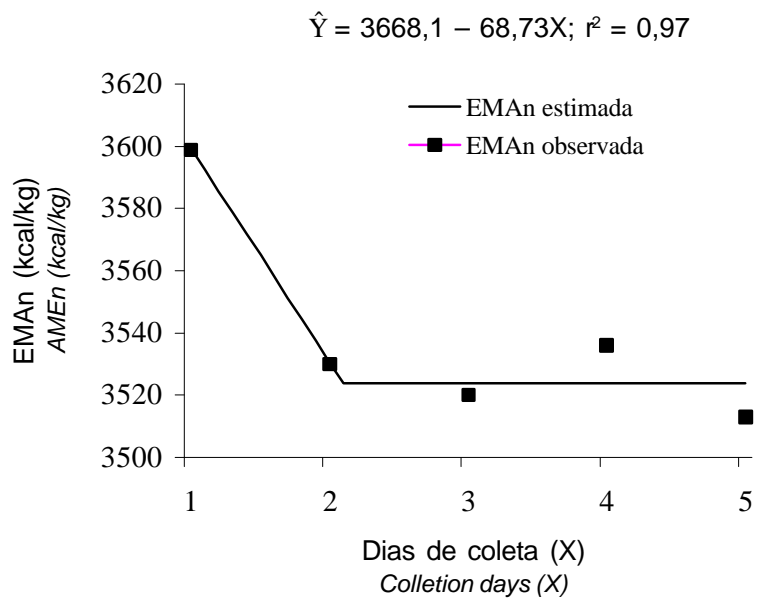

Figura 1 - Energia metabolizável aparente corrigida pelo nitrogênio retido (EMAn) de rações à base de milho e farelo de soja, determinada pelo método de coleta total de excretas, segundo os dias de coleta.

Figure 1 - Apparent metabolizable energy corrected by retained nitrogen (AMEn) of corn and soybean meal based diet, determinated by the total excreta collection, according to collection days.

é trabalhoso e, conseqüentemente, de maior custo. Entretanto, os resultados obtidos neste estudo sugerem que este período pode ser reduzido para três dias, sem comprometer a precisão das estimativas ou ocasionar alterações significativas nos valores determinados com mais de dois dias de coleta. Vale ressaltar, 


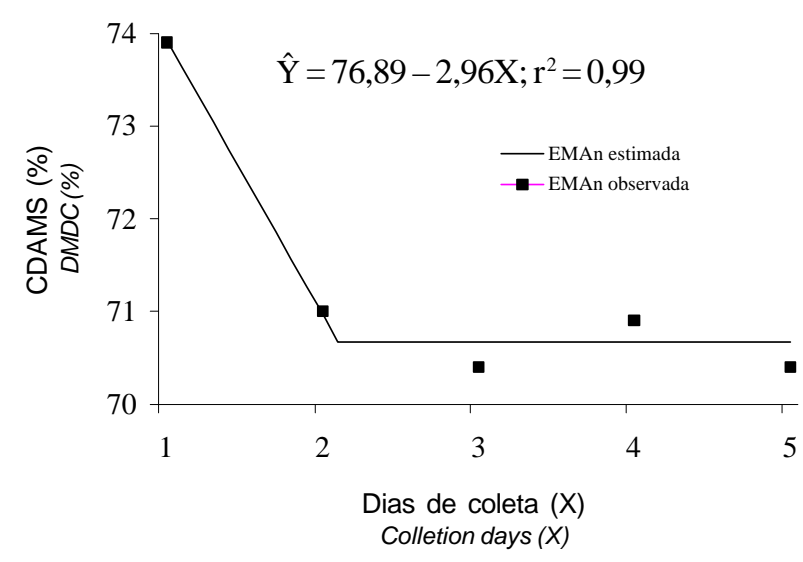

Figura 2 - Digestibilidade aparente da matéria seca de rações à base de milho e farelo de soja (CDAMS), determinada pelo método de coleta total de excretas, segundo os dias de coleta.

Figure 2 - Apparent dry matter digestibility coefficients (DMDC) of corn and soybean meal based diet, determinated by the total excreta collection, according to collection days.

porém, que esses valores foram determinados para rações à base de milho e farelo de soja e não se trabalhou com determinações para alimentos. Acredita-se, no entanto, que estas informações podem ser extrapoladas para valores energéticos de alimentos específicos, mas trabalhos que venham confirmar essas suposições são necessários.

Os valores médios dos CDAMS e EMAn determinados pela metodologia em que se utilizou o óxido crômico como indicador fecal estão apresentados nas Tabelas 3 e 4, respectivamente. Como observado no método de coleta total, o uso de períodos consecutivos de coleta não influenciou $(\mathrm{P}>0,05)$ os valores obtidos.
Houve interação $(\mathrm{P}<0,05)$ do período de coleta e dos níveis de óxido crômico utilizados sobre os valores de CDAMS e EMAn. Observou-se que, de maneira geral, houve certa inconstância nos valores determinados, independentemente de ter-se avaliado os níveis de cromo em cada tempo de coleta ou vice-versa, obtendo-se respostas linear, quadrática ou cúbica ( $\mathrm{P}<0,05)$, tanto dos tempos de coleta em cada nível de cromo utilizado na ração quanto dos níveis de cromo em cada tempo de coleta.

Portanto, os resultados obtidos evidenciam as variações nos valores de EMAn e CDAMS quando a metodologia com óxido crômico é utilizada. Como não foi de interesse prático definir um nível de cromo para cada tempo de coleta ou tempo de coleta para cada nível de cromo utilizado na ração, estas indicam que a metodologia do óxido crômico como indicador deve ser usada com certa cautela em ensaios nos quais se pretende determinar valores energéticos ou digestibilidade aparente de nutrientes, cujos resultados podem variar em função do nível de cromo e do tempo adotados para coleta de excretas.

De maneira geral, menores erros-padrão das estimativas foram observados quando se utilizou três dias de coleta, até o nível de $0,6 \%$ de inclusão do indicador óxido crômico. Por outro lado, quando se utilizou o nível de $0,8 \%$, foi necessário tempo maior de coleta, sugerindo que, quando se utiliza nível mais alto de óxido crômico na ração, devem ser adotados períodos mais longos de coleta, possivelmente em razão de sua passagem mais lenta pelo trato digestório (TD) em virtude da alta concentração. Também pode ter ocorrido recuperação incompleta do indicador nas excretas, talvez pelo seu acúmulo, em alguma parte do TD do animal quando níveis superiores a $0,6 \%$ foram

Tabela 3 - Coeficientes de digestibilidade aparente da matéria seca (CDAMS), obtidos com o uso do óxido crômico como indicador, e seus respectivos erros-padrão

Table 3 - Apparent dry matter digestibility coefficients (DMDC) obtained with chromium oxide indicator and respective standard errors

\begin{tabular}{lcccc}
\hline $\begin{array}{l}\text { Tempo de coleta } \\
\text { Collection period }\end{array}$ & \multicolumn{4}{c}{$\begin{array}{c}\text { Óxido crômico }(\%) \pm(\mathrm{EP}) \\
\text { Chromium oxide }(\%) \pm(\text { standard errors })\end{array}$} \\
\cline { 2 - 5 } & $0,2^{1}$ & $0,4^{3}$ & $0,6^{2}$ & $0,8^{3}$ \\
\hline $1 \operatorname{dia}^{2}($ day) & $79,31(0,40)$ & $76,80(0,33)$ & $74,09(0,58)$ & $68,03(1,17)$ \\
$2 \operatorname{dias}^{3}($ days) & $78,25(0,35)$ & $74,99(0,32)$ & $70,25(0,84)$ & $74,48(0,24)$ \\
$3 \operatorname{dias}^{2}($ days) & $77,21(0,30)$ & $75,40(0,09)$ & $71,44(0,27)$ & $73,40(0,34)$ \\
$4 \operatorname{dias}^{3}$ (days) & $76,38(0,38)$ & $75,20(0,22)$ & $71,16(0,49)$ & $75,56(0,12)$ \\
$5 \operatorname{dias}^{2}$ (days) & $76,72(0,48)$ & $74,17(0,11)$ & $74,99(0,42)$ & $76,31(0,10)$ \\
\hline
\end{tabular}

\footnotetext{
${ }_{1}^{1}$ Resposta linear (Linear response).

2 Resposta quadrática (Quadratic response).

${ }^{3}$ Resposta cúbica (Cubic response).
} 
Tabela 4 - Valores de energia metabolizável aparente corrigida pelo nitrogênio retido (EMAn - kcal/kg de MS), obtidos com o indicador óxido crômico, e seus respectivos erros-padrão (EP)

Table 4 - Apparent metabolizable energy corrected by the retained nitrogen values (AMEn - kcal/kg of DM) obtained with the chromium oxide indicator and the respective standard errors

Tempo de coleta

Óxido crômico $(\%) \pm(\mathrm{EP})$

Collection period

Chromium oxide (\%) \pm (standard errors)

\begin{tabular}{lllll}
\cline { 2 - 5 } & \multicolumn{1}{c}{$0,2^{1}$} & \multicolumn{1}{c}{$0,4^{3}$} & \multicolumn{1}{c}{$0,6^{2}$} & \multicolumn{1}{c}{$0,8^{3}$} \\
\hline $1 \operatorname{dia}^{2}($ day) & $3785(17)$ & $3774(9)$ & $3617(20)$ & $3413(36)$ \\
$2 \operatorname{dias}^{3}($ days) & $3763(12)$ & $3725(14)$ & $3478(27)$ & $3631(8)$ \\
$3 \operatorname{dias}^{3}(\text { days })^{2}$ & $3735(8)$ & $3740(6)$ & $3544(8)$ & $3589(16)$ \\
$4 \operatorname{dias}^{3}($ days) & $3717(10)$ & $3734(10)$ & $3535(16)$ & $3678(9)$ \\
$5 \operatorname{dias}^{2}$ days $^{2}$ & $3719(18)$ & $3692(10)$ & $3646(11)$ & $3713(1)$ \\
\hline
\end{tabular}

${ }_{1}^{1}$ Resposta linear (Linear response).

2 Resposta quadrática (Quadratic response).

${ }^{3}$ Resposta cúbica (Cubic response).

utilizados. Ressalta-se, portanto, que nenhum procedimento estatístico foi empregado na avaliação das estimativas com relação ao seu erro-padrão médio, fazendose apenas inferências com base no valor absoluto determinado. Desse modo, os dados obtidos sugerem que pelo menos três dias de coleta podem ser suficientes, nas duas metodologias, para se obter valores aceitáveis de CDAMS e EMAn. Uma diminuição gradativa dos errospadrão, à medida que se aumentou o tempo de coleta, foi observada por Fialho et al. (1985), ao trabalharem com suínos e utilizarem o óxido férrico como marcador, e também por Sibbald \& Price (1975), com aves, os quais encontraram menores erros-padrão das estimativas para tempos maiores de coleta. Todavia, neste trabalho, os resultados de CDAMS e EMAn encontrados mostraram valores absolutos dos erros-padrão variáveis, em relação ao tempo de coleta e ao nível de óxido crômico na ração.

Considerando-se que o método de coleta total de excretas é o método comumente utilizado na determinação dos valores energéticos dos alimentos, e como a resposta para os valores obtidos em relação ao tempo de coleta mostrou um platô superior a dois dias, adotou-se o período de três dias de coleta, cujos valores absolutos dos erros-padrão da média foram aparentemente menores, como ideal para se comparar as duas metodologias. Consta, nas figuras 3 e 4, a comparação entre os valores de CDAMS e EMAn, obtidos com as duas metodologias e com três dias de coleta de excretas. Pelas figuras apresentadas, sugere-se que $0,6 \%$ é o nível de inclusão de óxido crômico que possibilita determinar valores semelhantes de CDAMS e EMAn nas duas metodologias.

$\mathrm{Na}$ Figura 5, são apresentados os resultados de um estudo comparativo entre as duas metodologias, utilizando-se três dias de coleta, em que se calculou a razão entre os valores de EMAn e dos CDAMS determinados com o uso do indicador óxido crômico e aqueles determinados pelo método de coleta total de excretas (3.520 kcal de EMAn/kg de MS e 70,4\% de digestibilidade da MS, respectivamente). Pelos resultados, observou-se que os níveis que possibilitaram determinar valores mais próximos de EMAn e CDAMS foram, respectivamente, 0,665 e 0,667\% de inclusão

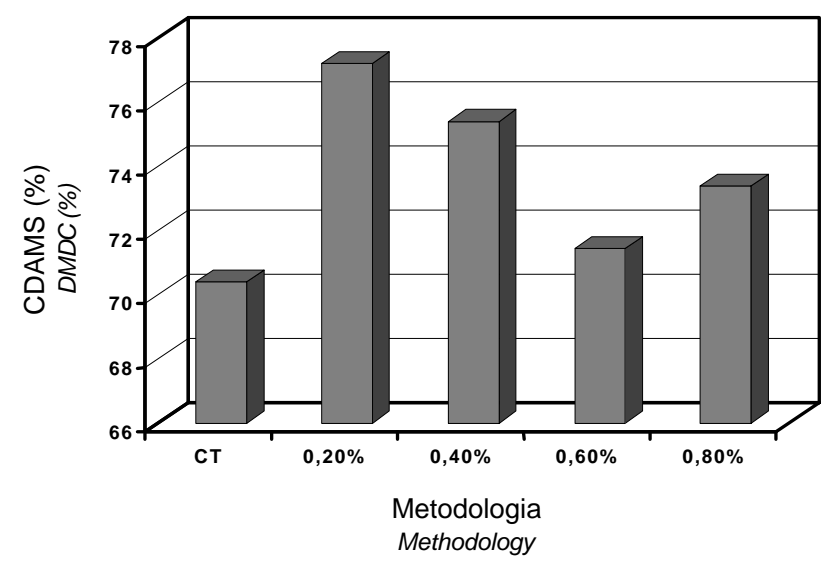

Figura 3 - Comparação entre os valores dos coeficientes de digestibilidade aparente da matéria seca (CDAMS), obtidos pelo método de coleta total de excretas (CT) e pelo indicador óxido crômico, nos diferentes níveis de inclusão, para três dias de coleta.

Figure 3 - Comparison of the dry matter aparent digestibility coefficients (DMDC) values obtained in the total excreta collection and chromium oxide as indicator, in the different levels of inclusion, for the three days of collection. 


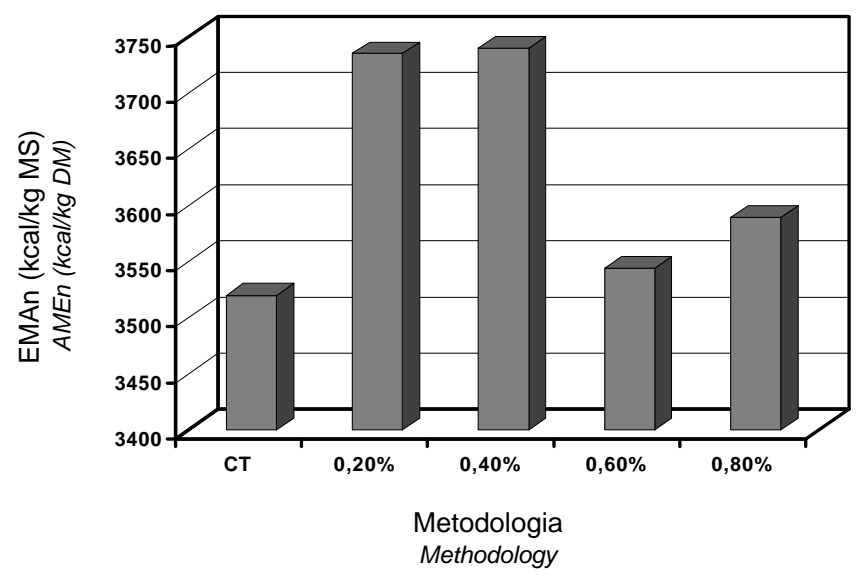

Figura 4 - Comparação dos valores de energia metabolizável aparente corrigida pelo nitrogênio retido (EMAn), obtidos pelo método de coleta total de excretas (CT) e pelo indicador óxido crômico, nos diferentes níveis de inclusão, para três dias de coleta.

Figure 4 - Comparison of the apparent metabolizable energy corrected by the nitrogen retained (AMEn) obtained in the total excreta collection and chromium oxide as indicator in the different levels of inclusion for the three days of collection.

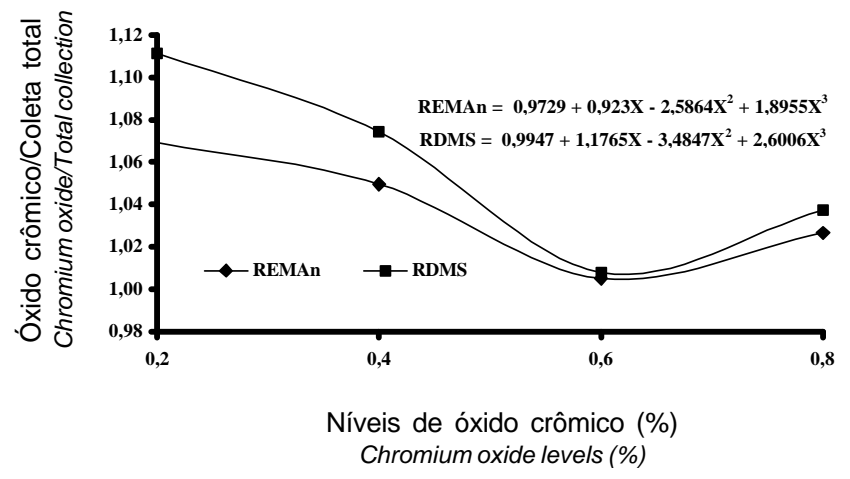

Figura 5 - Razão entre os valores de EMAn (REMAn) e CDAMS (RDMS) obtidos com o indicador óxido crômico e aqueles encontrados com a metodologia de coleta total de excretas, utilizando-se três dias de coleta.

Figure 5 - Metabolizable energy values ratio (AMEnR) and dry matter digestibility coefficients ratio (DMDCR) obtained with the chromium oxide indicator and those obtained with total excreta collection methodology, using three days of collection.

do indicador, correspondendo a uma razão de 1,0003 e 1,0008. Ou seja, com o uso destes níveis de óxido crômico, as duas metodologias praticamente se equivaleram na estimativa dos valores de EMAn e CDAMS. Porém, considerando-se a importância de se padronizar as metodologias de determinação do valor nutricional dos alimentos, principalmente dos valores energéticos, ressalta-se a necessidade de novos estudos que venham a confirmar estes resultados utilizando-se três dias de coleta de excretas e nível de $0,665 \%$ de óxido crômico como indicador na ração.

\section{Conclusões}

O uso de três dias de coleta é suficiente, na metodologia de coleta total de excretas, para se determinar valores de CDAMS e EMAn de rações à base de milho e farelo de soja. Adotando-se três dias de coleta e $0,665 \%$ de óxido crômico na ração, é possível determinar valores de EMAn e CDAMS semelhantes aos obtidos pelo método tradicional de coleta total de excretas.

\section{Literatura Citada}

ALBINO, L.T.F.; ROSTAGNO, H.S.; TAFURI, M.L. et al. Determinação dos valores de energia metabolizável aparente e verdadeira de alguns alimentos para aves, usando diferentes métodos. Revista da Sociedade Brasileira de Zootecnia, v.21, n.6, p.1047-1058, 1992

ASSOCIATION OF OFFICIAL ANALYTICAL CHEMIST AOAC. Official methods of analysis: agricultural chemicals, contaminants and drogs. 15.ed. Washington: Association of Official Analytical Chemists, 1990. v.1, 684p.

BRAGA, J.M. Avaliação da fertilidade do solo: ensaios de campo. Viçosa, MG: Universidade Federal de Viçosa, 1983. 101p.

FARRELL, D.J. An assessment of quick bioassays for determinating the true metabolizable energy and apparent metabolizable energy of poultry feedstuffs. World's Poultry Science, v.37, n.2, p.72-83, 1981.

FARRELL, D.J. Rapid determination of metabolizable energy of foods using cokerels. British Poultry Science, v.19, n.3, p.303-308, 1978.

FIALHO, E.T.; BELLAVER, C.; FREITAS, A.R. et al. Influência da substituição do milho e do farelo de soja no balanço protéico e energético em suínos. Pesquisa Agropecuária Brasileira, v.20, n.10, p.1229-1236, 1985.

FERREIRA, D.F. Sistema de análise estatística para dados balanceados (SISVAR). Lavras: Universidade Federal de Lavras/DEX. 2000.

FURLAN, A.C.; ANDREOTTI, M.O.; MURAKAMI, A.E. et al. Valores energéticos de alguns alimentos determinados com codornas japonesas (Coturnix coturnix japonica). Revista Brasileira de Zootecnia, v.27, n.6, p.1147-1150, 1998.

HARTEL, H. Influence of food input and procedure of determination on metabolizable energy and digestibility of a diet measured with young and adult birds. British Poultry Science, n.1, p.11-39, 1986.

KESSLER, J. W.; THOMAS, O. P. The effect of cecectomy and extension of the collection period on the true metabolizable energy values of soybean meal, feather meal, fish meal and blood meal. Poultry Science, v.60, n.12, p.2639-2647, 1981. 
MATTERSON, L.D.; POTTER, L.M.; STUTZ, M.W. et al. The metabolizable energy of feed ingredients for chickens. Storrs: University of Connecticut, Agricultural Experiment Station, 1965. 11p (Research Report, 7).

McNAB, J.M. Factors affecting the energy value of wheat for poultry. World's Poultry Science Journal, v.52, n.1, p.69-73, 1996.

NASCIMENTO, A.H.; GOMES, P.C.; ALBINO, L.F.T. et al. Valores de composição química e energética de alimentos para frangos de corte. Revista Brasileira de Zootecnia, v.27, n.3, p.579-583, 1998.

PARSONS, C.M.; POTTER, L.M.; BLISS, B.A. True metabolizable energy corrected to nitrogen equilibrium. Poultry Science, v.61, n.11, p.2241-2246, 1982.

POTTER, L.M. The precision of measuring metabolizable energy in poultry feedstuffs. Feedstuffs, v.44, n.12, p.28-30,1972.

RODRIGUES, P.B.; ROSTAGNO, H.S.; ALBINO, L.F.T. et al. Valores energéticos da soja e subprodutos da soja, determinados com galos adultos cecectomizados. Revista Brasileira de Zootecnia, v.31, n.4, p.1771-1782, 2002.

RODRIGUES, P.B.; ROSTAGNO, H.S.; ALBINO, L.F.T. et al. Valores energéticos do milheto, do milho e subprodutos do milho, determinados com frangos de corte e galos adultos. Revista Brasileira de Zootecnia, v.30, n.6, p.1767-1778, 2001.

ROSTAGNO, H.S.; ALBINO, L.F.T.; DONZELLE, J.L. et al. Tabelas brasileiras para aves e suínos: composição de alimentos e exigências nutricionais. Viçosa, MG: Universidade Federal de Viçosa, 2000. 141p.

SCHANG, M.J.; HAMILTON, R.M.G. Comparison of two bioassays using adults cocks and four indirect methods for estimating the metabolizable energy content of diferent feedingstuffs. Poultry Science, v.61, p.1344-1353, 1982.
SIBBALD, I.R.; PRICE, K. Variation in the metabolizable energy values of diets and dietary components feed to adult roosters. Poultry Science, v.54, n.2, 448-56, 1975.

SIBBALD, I.R. A bioaasay for the true metabolizable energy in feedstuffs. Poultry Science, v.55, n.1, p.303-308, 1976.

SIBBALD, I.R.; SLINGER, S.J. A biological assay for metabolizable energy in poultry feed ingredients together with findings which demonstrate some of the problems associated with the evaluation of fats. Poultry Science, v.42, n.2, p.313-325,1963.

SIBBALD, I.R.; SUMMERS, I.D.; SLINGER, S.J. Factores affecting the metabolizable energy content poultry feedstuffs. Poultry Science, v.44, p.544-556, 1960.

SILVA, D.J. Análise de alimentos (métodos químicos e biológicos). 2.ed. Viçosa, MG: Universidade Federal de Viçosa, 1990, 165p.

TENESACA, L.G.; SELL, J.L. Influence of na indigestible material on energy excretion by roosters and on true metabolizable energy of corn. Poultry Science, v.60, n.3, p.623-630, 1981.

VIEITES, F.M.; ALBINO, L.F.T., SOARES, P.R. et al. Valores de energia metabolizável aparente da farinha de carne e ossos para aves. Revista Brasileira de Zootecnia, v.29, n.6 (Supl. 2), p.2292-2299, 1998

ZELENKA, J. Effects of sex, age and food intake upon metabolizable energy values in broiler chickens. British Journal of Nutrition, v.38, n.3, p.281-284, 1997.

Recebido em: 30/04/03

Aceito em: 23/03/05 